\title{
Minimal Invasive Technique in Atrial Septal Defect Surgery
}

\author{
Feridoun Sabzi $^{\mathrm{a}}$, Reza Faraji ${ }^{\mathrm{a}}$, Mahmood Kazeminasab ${ }^{\mathrm{b}, \mathrm{c}}$
}

\begin{abstract}
Background: Median sternotomy with minimal skin incision (MSWMSI) and modified anterior mini-thoracotomy (MAMT) approach that both are innovative techniques modified from previous documented techniques are important alternative to conventional median sternotomy in atrial septal defect (ASD) repair. Our aim is to explain the details of two performed techniques in our center and explain the results.
\end{abstract}

Methods: Totally 54 children with ASD (20 female and 34 male) were operated with two different techniques i.e. MAMT and MSWMSI in Imam Ali heart surgery center between May 2010 and May 2013. Intra and postoperative variables such as cardiopulmonary bypass time and aortic cross-clamp time, intensive care unit stay time, length of incision, postoperative hematoma and seroma, dehiscence mortality, exploration for postoperative bleeding, neurologic complication, infection and amount of blood transfusion were recorded.

Results: Mean cardiopulmonary bypass time was $30 \pm 11 \mathrm{~min}$, and mean aortic cross-clamp time was $7 \pm 2 \mathrm{~min}$. The mean amount of blood transfusion was $150 \pm 39 \mathrm{~mL}$, and the mean chest tube drainage after surgery was $140 \pm 57 \mathrm{~mL}$. Superficial skin infection occurred in three patients. Subcutaneous hematoma and seroma were founded in six patients. In 50 cases the defect was secundum type, in two patients it was sinus venosus type, and in two with associated perimembranous ventricular septal defect repair.

Conclusion: Both approaches are safe and may be the surgical techniques of choice for secundum ASD repair in all age groups; and we can also utilize these techniques for more complicated kinds of surgery, for instance, sinus venosus type ASD with or without partial anomalous defect.

Keywords: Minimally invasive surgical procedures; Sternotomy; Heart defects

Manuscript submitted February 27, 2018, accepted March 12, 2018

aKermanshah Cardiovascular Research Center, Kermanshah University of Medical Sciences, Kermanshah, Iran

${ }^{b}$ Student Research Committee, Shahid Sadoughi University of Medical Sciences, Yazd, Iran

${ }^{\mathrm{c} C o r r e s p o n d i n g ~ A u t h o r: ~ M a h m o o d ~ K a z e m i n a s a b, ~ S t u d e n t ~ R e s e a r c h ~ C o m m i t-~}$ tee, Shahid Sadoughi University of Medical Sciences, Yazd, Iran.

Email: info.kazeminasab@gmail.com

doi: https://doi.org/10.14740/cr699w

\section{Introduction}

Atrial septal defect (ASD) is a common congenital anomaly that requires intervention in children in almost all patients. Percutaneous closure of ASD with Amplatzer is widespreadly used in tertiary center with good facility and experienced cardiologist for implantation of this device. However use of this technique is limited by its high cost and its charge uncovering by insurance company. In other hand, risks of emboli and residual ASD, high cost and its thrombotic complications caused by the device make it not used in small center that had not good facility to handle its post-interventional complications [1]. In the conventional form of surgery, midline sternotomy and use of total cardiopulmonary bypass (CBP) with ascending aorta and superior vena cava (SVC) and inferior vena cava (IVC) cannulation is performed. Sometimes residual ASD post device implantation needs to be closed by open cardiac surgery in young children; however, little is known about the safety and outcome of this procedure in infants [2]. In this study, technical safety of secundum ASD closure with these two different approaches i.e. modified anterior mini-thoracotomy (MAMT) (in thin children) and median sternotomy with minimal skin incision (MSWMSI) (in fat children) were evaluated. When the trends of the parents of these patients were for minimal incisions, we would choose a technique with respect to size of the child. Surgical techniques, our experiences and results are discussed in details.

\section{Materials and Methods}

From 2007 to 2012 two different surgical approaches were studied with mean incision length of $7 \pm 2.5 \mathrm{~cm}$ in 54 patients (24 male and 20 female). All of the children had isolated ASD with no coexistence of another congenital disease. All of the patients had important left-to-right shunt, and neurologic deficit was not observed preoperatively. The pulmonary arterial pressure was evaluated in all patients preoperatively. Intra and post operative variables such as cardiopulmonary bypass time and aortic cross-clamp time, intensive care unit stay time, length of incision, postoperative hematoma and seroma, dehiscence mortality, exploration for postoperative bleeding, neurologic complication, infection and amount of blood transfusion were recorded in both of the two techniques.

\section{Surgical method}

In first technique of MSWMSI (used in thin children), left lung 
endotracheal intubation was performed and a small round pad was put under right shoulder of patients with about 30 degree elevation for better exposure. The small skin incision in the fifth right anterior intercostal space, exactly in the mid clavicular line was performed with small retractor for rib spreading. Sometimes in some patients ascending aorta was not accessible to cannulation in the fifth intercostals space, otherwise we dislocated costo-sternal junction in upper rib to achieve the best exposure to ascending aorta. In this modified technique in case of difficult accessing to aorta in spite of violating upper or lower intercostals space as mentioned in other studies, we dislocated upper or lower costo-sternal cartilage. After we assured the exact position of aorta and easy accessing to cannulation, the incision was expanded. A rib retractor has been employed and the right lung retracted posteriorly with a wet gaze. Pericardium was opened carefully in manner that phrenic nerve was not injured and in all cases total or partial thymectomy was performed for better exposure, then we divided a piece of pericardium for possible closure of ASD, and then with the stay sutures pericardium retracted and hanged to retractor blades for better exposure of aorta and SVC. Central cannulations of aorta were performed in all and then the SVC was cannulated and a tape putted around it and then snuggled. IVC cannulation in this position needs upward pulling of heart that will cause severe hypotension and arrhythmia and rarely ventricular fibrillation. With regards to these complications, after institution of CPB, upward drawing of heart caused no severe hemodynamic change and easily access to IVC, and IVC was obtained in all patients. However pericardial retraction sutures gave good access to aorta, but better exposure could be obtained by downward traction on aorta by a small mosquito clump. After beginning of CPB, conventional cardiac surgery device were used, and by aortic cross-clamping and cardioplegin injection right atrium was opened and ASD was repaired directly. Ascending aorta was cross-clamped with simple aortic clamp from thoracotomy incision in all of the cases. We have not used a DeBakey type trans-thoracic aortic clamp by violating the third inter-costal space. De-airing of left atrium was performed via the last upper sutures of ASD closure, and de-airing of left ventricle was achieved by cardioplegia line connected to vent. By high frequency mediastinal shaking, adhered airs bubbles to left ventricular papillary muscles or internal ventricle wall were released and vented. After de-clamping of aorta and weaning of $\mathrm{CPB}$, de-cannulation, administration of protamine, hemostasis, and chest tube insertion, the incision can be closed in three layers i.e. ribs suture, two muscular layers and subcutaneous stitches and skin closure.

The second approach or MAMT technique that was used by author is an innovation technique that was selectively performed in our center. A mini-incision ( $4-5 \mathrm{~cm}$ ) was performed in the central part of the skin on sternum. Then the subcutaneous tissue was released from the superior, inferior and lateral side of incision, and full sternotomy is performed. After central aortic cannulation and cannulating of the SVC with right angled cannula and with a tap around it, the extracorporeal circulation was instituted, the heart pulled upward, and access to the IVC would be possible after beginning of CBP and stopping ventilation in this small incision. Also we surround IVC with tape after starting of CPB. After bi-cava cannulation the

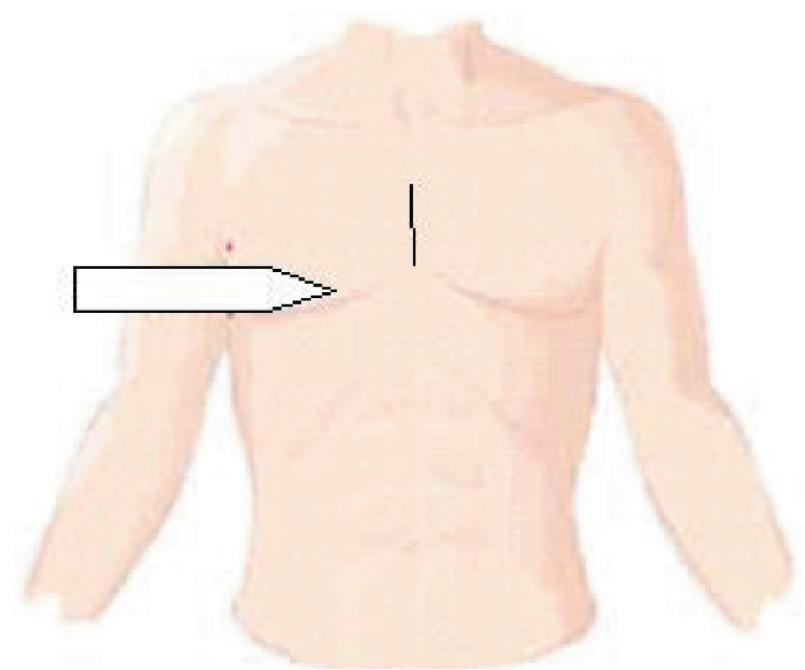

Figure 1. White arrow shows limited mid sternotomy incision.

operation is advanced through cardioplegia cardiac arrest. This method is previously used by mini-incision in inferior part of sternum and lower sternotomy. But our technique by mini-incision in central part of sternum is an innovation and was not performed previously (Fig. 1) [3-6]. It should be noted that in our study conventional sternal retractor was used, and the femoral artery was not cannulated in any patient. Careful literature search revealed that subtypes of minimal invasive techniques for ASD repair in children (lateral thoracotomy, anterolateral thoracotomy, lower sternotomy by minimal invasive technique) need institution of CPB by peripheral cannulation of femoral artery, cervical cannulation of SVC and femoral venous cannulation for IVC that were discussed by some authors. The complicated cervical technique of SVC cannulation needs advanced imaging technology in operating room, expert and educated anesthesiologist and radiologist and sometimes negative pressure pump reservoir. This facility along with special cannula did not exist in our center.

\section{Results}

After the operation, the transthoracic echocardiography follow-up revealed no residual postoperative ASD in all of the patients. In all of the 54 patients, ASD was closed directly without a pericardial patch usage. The mean aortic clamp time and CPB time were $7 \pm 2$ and $30 \pm 11 \mathrm{~min}$, respectively. The TEE was not used in patients after the operation. The temporary left or right bundle branch block in five patients was monitored after the operation and was recovered spontaneously after 2 - 7 days. No sternal dehiscence was observed in all patients. Superficial infection of mid sternotomy was observed in five patients who were treated by oral antibiotics. In MAMT technique, seroma and blood things hematoma were formed in sternotomy skin flap which was drained with a syringe in six patients. No neurologic complication was observed. The mean ventilation time was $10 \pm 3 \mathrm{~h}$ and patients were transferred to the ward from ICU after $25 \mathrm{~h}$. All of the patients and their 
parents were satisfied by mini-incision especially in MSWMSI technique.

\section{Discussion}

One of the important complications of conventional midline sternotomy in children is the psychological disorders caused by skin incision sequels in midline sternotomy. To reduce this psychological complication, two different minimal invasive techniques i.e. MSWMSI and MAMT were used. To institute extracorporeal circulation central aortic and bi-caval cannulation was inserted; cardiac arrest was settled by aortic root cannulation and cardioplegin injection. These two approaches were accomplished to reduce the psychological complications of skin incision in ASD, and for this aim minimal skin incision has been used more commonly in cardiac center. The main step in CPB establishment in ASD operation is the arterial cannulation, (aortic or femoral) and IVC cannulation. In our center, due to absence of facility, method such as SVC cannulation using Seldinger method is not applicable. In opposed to others studies in both methods, femoral cannulation was not used [7]. We believed that in both methods, despite limited operation's field view, by downward pulling of the aorta and use of DLP right angled cannula, the aortic cannulation is accessible; while in others' study, femoral artery was cannulated in all or some cases. In this study, our group had performed aortic cannulation in 53 patients without any surgical complication. The only problem was aortic cannula kinking by superior angle of the skin incision, but the use of spiral DLP cannula has solved this problem. After aortic and SVC cannulation and cardioplegia cannula insertion, the $\mathrm{CPB}$ was established; and then, by upward pulling of the heart, the IVC is cannulated. Without the CPB institution, the IVC access through this small incision by upward dislocation of heart will cause severe hypotension and arrhythmia [8].

According to our experience, aortic cannulation is safe and easy, and traumatic complications of femoral artery cannulation as dissection which is typically common in infants, is absent here. In this paper, right parasternal method was not used due to the possibility of the superior and inferior ribs fractures by spreading of retractor and injury to internal mammary artery. One of the common dermal complications, by these two techniques is tension on the skin and the skin edges necrosis which is specially observed in superior and inferior angle of incision, and needs debridement at the end of the operation [9]. In MSWMSI unlike the classic incision, in order to cannulate the aorta it is needed to displace costo-sternal junction of upper or lower rib and sometimes the mammary internal artery is sacrificed. To reduce operation time and difficulty in patch repair of ASD in small operation field, defect in all of the patients were repaired by direct method. No postoperative low cardiac output or pulmonary edema or suture dehiscence was observed in this method. To protect the heart, cardioplegia injected in aortic root and the fibrillation method was not used. Another problem is de-airing of heart chambers, in opposed to complete midline sternotomy, upward lifting of heart is impossible for deaeration of left ventricle at apex in these small incisions. For this aim, we used the mediastinal shaking and car- dioplegia cannula as a vent. No neurologic complication was observed regards to residual air bubbles in heart chambers. We believe that cardiac arrest with cardioplegia in minimal skin incision is easier for ASD operation and it does not have complications of ventricular fibrillation like segmental hypokinesia and cardiac-isoenzyme changes. We used these approaches for ASD repair based on the patients' body physique after evaluating different minimal invasive methods like L-type or reverse $\mathrm{T}$ sternotomy or parasternal methods. If the patient is slim and there is enough space between the ribs, MSWMSI was used and in the case of obese patient and older than 10 years, MATMA was used. Aortic cannulation in MSWMSI is easier than MATMA but skin incision is longer $(1-2 \mathrm{~cm})$ than that in technique MATMA. The main step is aortic cannulation which should be performed carefully and needs skills in using the right angled aortic cannula. Separating costosternal junction of upper or lower rib in thoracotomy incision makes easy access to the aorta. In opposed to other studies we did not use TEE for monitoring the cardiac deairing process. By using mediastinal shaking and aortic root venting de-airing of heart chambers was performed; and we did not observe any neurologic complication in all operated patients.

\section{Conclusions}

These two methods were used in 53 patients without any major complication. In addition to pretty appearance of incision which is important for children, the ICU and ward stay was shorter and traumatic injury of femoral cannulation was not observed. In MATMA technique, the aortic cannulation is more difficult, but the incision site is more desired cosmetically. Meanwhile in MATMA technique the aortic cannulation is easier, although despite small length of incision it is less pleased by children and their parents.

\section{Grant Support}

None.

\section{Conflict of Interest}

All the authors have reported no conflict of interest.

\section{References}

1. Agarwal SK, Ghosh PK, Mittal PK. Failure of devices used for closure of atrial septal defects: mechanisms and management. J Thorac Cardiovasc Surg. 1996;112(1):2126.

2. Lancaster LL, Mavroudis C, Rees AH, Slater AD, Ganzel BL, Gray LA, Jr. Surgical approach to atrial septal defect in the female. Right thoracotomy versus sternotomy. Am Surg. 1990;56(4):218-221.

3. https://www.hopkinsmedicine.org/heart_vascular_insti- 
tute/conditions treatments/treatments/minimally invasive_atrial_septal_defect_closure.html.

4. Diab KA, Cao QL, Bacha EA, Hijazi ZM. Device closure of atrial septal defects with the Amplatzer septal occluder: safety and outcome in infants. J Thorac Cardiovasc Surg. 2007;134(4):960-966.

5. Berger F, Vogel M, Alexi-Meskishvili V, Lange PE. Comparison of results and complications of surgical and Amplatzer device closure of atrial septal defects. J Thorac Cardiovasc Surg. 1999;118(4):674-678; discussion 678680.

6. Bennhagen RG, McLaughlin P, Benson LN. Contemporary management of children with atrial septal defects: a focus on transcatheter closure. Am J Cardiovasc Drugs.
2001;1(6):445-454.

7. Chang CH, Lin PJ, Chu JJ, Liu HP, Tsai FC, Chung YY, Kung CC, et al. Surgical closure of atrial septal defect. Minimally invasive cardiac surgery or median sternotomy? Surg Endosc. 1998;12(6):820-824.

8. Mishra S, Tomar M, Malhotra R, Radhakrishnan S, Mishra Y, Iyer KS, Shrivastava S, et al. Comparison between transcatheter closure and minimally invasive surgery for fossa ovalis atrial septal defect: a single institutional experience. Indian Heart J. 2008;60(2):125-132.

9. Izzat MB, Yim AP, El-Zufari MH. Limited access atrial septal defect closure and the evolution of minimally invasive surgery. Ann Thorac Cardiovasc Surg. 1998;4(2):5658 . 